\title{
Personnes Âgées et Réanimation
}

\section{Bertrand Guidet, Antonio Artigas. Springer-Verlag France, Paris 2012. ISBN 978-2-8178-0286-2}

\author{
Alain Deschamps, MD, PhD
}

Received: 14 May 2012/ Accepted: 24 May 2012/Published online: 22 June 2012

(C) Canadian Anesthesiologists' Society 2012

Qu'avons-nous à apprendre à propos des patients gériatriques aux soins intensifs? À peu près tout. C'est le défi que nous décrit de façon détaillée le livre de référence «Personnes âgées et réanimation » édité par Bertrand Guidet et Antonio Artigas. Dès l'introduction, on met cartes sur table: l'approche devra être multidisciplinaire et c'est dans cet esprit que les chapitres se succèdent touchant aux aspects spécifiques de la «géronto-réanimation ».

Le premier chapitre nous fait part de l'évolution démographique en Europe, où on peut démontrer facilement le vieillissement de la population. Cependant, tout comme dans les autres continents d'ailleurs, ce vieillissement n'est pas uniforme, certains pays étant plus touchés que d'autres. Il y aura 24,4 millions de personnes âgées de 85 ans ou plus dans l'Union Européenne en 2040 (plus du double de 2010) avec la plus grande augmentation proportionnelle en Pologne (plus du triple), et ce, principalement à cause de la diminution de la mortalité.

Comment définir la vieillesse? Quiconque a prodigué des soins de santé comprend immédiatement la différence entre l'âge chronologique et l'âge biologique. Cette différence, qu'elle soit d'origine génétique ou environnementale, rend les systèmes de classification de la personne âgée très problématiques. Le deuxième chapitre met en évidence le défi de cette définition, donne espoir que des biomarqueurs pourront un jour aider, offre d'utiliser une échelle de fragilité pour définir l'âge biologique, et établit l'importance de l'expérience clinique et empirique lors du processus de décision.

Après ces deux premiers chapitres, qui offre au lecteur une image claire de l'ampleur du problème pour les

A. Deschamps, MD, PhD ( $\square)$

Institut de Cardiologie de Montréal, Montreal, QC, Canada

e-mail: a.deschamps@umontreal.ca décennies à venir, le reste du livre est consacré à l'état actuel de la disponibilité des soins intensifs pour les patients âgés, aux études qui seront nécessaires pour obtenir des données probantes à ce sujet, aux alternatives de traitement et à la pathophysiologie spécifique à cette population de patients. Parmi les sujets discutés, on retrouve: l'évaluation gériatrique standardisée, étape nécessaire et multidimensionnelle incluant le statut fonctionnel, cognitif, nutritionnel, spirituel, social et économique; les risques et bénéfices de l'admission aux soins intensifs et du besoin d'adapter les structures actuelles aux besoins de la personne âgée; l'importance de l'intervention d'une équipe médicale en préhospitalier pour évaluer le besoin de réanimation et orienter les patients âgés vers les urgences; les critères d'admission aux soins intensifs, incluant des études sur les facteurs prépondérants choisis par les intensivistes; les caractéristiques des séjours des patients âgés aux soins intensifs et les coûts associés; le processus décisionnel de limitation des soins; le pronostic immédiat et le pronostic prolongé selon les pathologies associées, avec distinction importante entre admission médicale et admission à la suite de chirurgie programmée; autonomie et qualité de vie après un séjour aux soins intensifs, en insistant sur une approche multidisciplinaire pour favoriser la réhabilitation et le retour aux activités normales; les dysfonctions neuromusculaires et la réduction des réserves organiques; l'aspect multidisciplinaire de la limitation des traitements et de la prise en charge de la fin de vie, incluant une section sur la responsabilité médicale, le devoir du médecin après la décision, l'accompagnement spirituel du patient et la prise en charge des familles; la recherche clinique chez les patients âgés, avec considérations méthodologiques et statistiques, incluant le haut taux de refus et la difficulté du consentement éclairé; la pharmacologie altérée chez le 
patient âgé; le patient âgé avec défaillance cardiaque aigue, en insistant sur les limites d' efficacité des médicaments disponibles dans cette population; un excellent survol de la réponse du système cardiovasculaire vieillissant au choc cardiogénique ou septique démontrant clairement l'impact négatif disproportionné du choc sur les organes vitaux; la diminution de la réponse immunitaire à la septicémie et les modifications dans les modalités de traitement; la défaillance respiratoire aigue, ses causes, la ventilation mécanique et le sevrage du respirateur; la place de la ventilation non invasive chez le patient âgé avec maladie pulmonaire obstructive chronique; les infections nocosomiales et le risque accru de morbidité chez le patient âgé; le pronostic de l'anémie et des transfusions sanguines, en soulignant les alternatives à la transfusion; les problèmes psychiatriques chez la personne âgée incluant les épisodes dépressifs majeurs, les troubles de la personnalité et l'évaluation du risque suicidaire; les soins intensifs neurologiques, incluant le vieillissement du cerveau, les accidents vasculaires cérébraux, les traumas crâniens, l'épilepsie et le pronostic de ces conditions; la prise en charge nutritionnelle chez le patient vieillissant; et enfin l'approche «evidence-based bundle » qui utilise les lettres ABCDE pour décrire les étapes d'évaluation des patients en ce qui concerne la sédation, le sevrage de la ventilation mécanique, le delirium, la mobilité précoce et l'exercice, avec les agents préconisés pour la sédation des patients âgés ainsi que le monitoring régulier pour le dépistage et le traitement du delirium.

Dans un ouvrage de référence il est impossible d'éviter un certain degré de répétition, surtout des données démographiques. Cependant, tous les chapitres font référence à de nombreuses études contemporaines dont les résultats sont discutés intelligemment en relation avec la pratique courante. De plus, la majorité des auteurs indiquent clairement les voies d'études futures pour l'amélioration la qualité des soins à la population âgée. L'abondance de tableaux et de figures offre un complément d'information qui peut être utilisé comme sommaire du sujet décrit. Les éditeurs ont intelligemment laissé les auteurs de certains chapitres s'exprimer en anglais, ce qui permet d'obtenir un compte rendu authentique de l'opinion de l'auteur.

Ce livre réussit à renseigner le lecteur, qu'il soit spécialisé ou non en gériatrie, sur les données actuelles au sujet des soins intensifs pour les patients âgés. Lorsque j'ai reçu les lignes directrices pour ce travail il était spécifié que «nous ne nous attendons pas à ce que vous lisiez le livre dans son intégralité », et bien franchement, je n'ai pu m'en empêcher.

Conflit d'intérêt Aucun. 